Collado Martínez, M.; Robles Tascón, J.A.; Álvarez del Palacio, E. (2021) Olympic Women in the International Descent of the Sella: Evolution of Participation. Revista Internacional de Medicina y Ciencias de la Actividad Física y el Deporte vol. 21 (81) pp. 99-116 Http://cdeporte.rediris.es/revista/revista81/artdescenso1220.htm

DOI: https://doi.org/10.15366/rimcafd2021.81.007

\title{
ORIGINAL
}

\section{MUJERES OLIMPICAS EN EL DESCENSO INTERNACIONAL DEL SELLA: EVOLUCIÓN DE SU PARTICIPACIÓN}

\section{OLYMPIC WOMEN IN THE INTERNATIONAL DESCENT OF THE SELLA: EVOLUTION OF PARTICIPATION}

\author{
Collado Martínez, M. ${ }^{1}$; Robles Tascón, J.A. ${ }^{2}$ y Álvarez del Palacio, E. ${ }^{2}$ \\ 1 Doctora en Ciencias de la Actividad Física y el Deporte. Profesora I.E.S Avelina Cerra. \\ Ribadesella. Asturias (España) mariacollado06@hotmail.com \\ ${ }^{2}$ Doctor en Ciencias de la Actividad Física y el Deporte. Profesor titular Facultad de Ciencias de \\ la Educación Física y del Deporte. Universidad de León. León (España) \\ jose.robles.tascon@unileon.es, eduardo.alvarez@unileon.es
}

Código UNESCO / UNESCO code: 5599 Otras Especialidades: Historia de la actividad física y el deporte

Clasificación Consejo de Europa / Council of Europe classification: 7. Historia del deporte / History of sport

Recibido 20 de febrero de 2019 Received February 20, 2019

Aceptado 22 de noviembre de 2019 Accepted November 22, 2019

\section{RESUMEN}

Este trabajo tiene como objetivo analizar el papel de la mujer en el Descenso Internacional del Sella, relacionándolo con la participación femenina en los juegos olímpicos. A continuación, nos hemos centrado en las palistas femeninas españolas, y su participación en la prueba deportiva que consiguió que se fundara la Federación Española de Piragüismo, el Descenso Internacional del Sella, gracias a su creador, Dionisio de la Huerta.

Utilizando una metodología cualitativa descriptiva multidisciplinar, hemos tenido en cuenta aspectos económicos, sociales, culturales y recreativos realizando un estudio de las palistas olímpicas, su palmarés, así como su participación en el Descenso Internacional del Sella. Los resultados muestran una incorporación tardía de la mujer al ámbito deportivo competitivo, ligado a estereotipos de género, así como desigualdades en las condiciones de participación. 
PALABRAS CLAVE Descenso del Sella, piragüismo, España, mujeres olímpicas mujer deporte.

\section{ABSTRACT}

This work aims to analyze the role of women in the International Descent of Sella, relating it to women's participation in the Olympic games. Next, we have focused on the Spanish female paddlers, and their participation in the sports event that enable the Spanish Canoeing Federation to be founded, the International Descent of the Sella, thanks to its creator, Dionisio de la Huerta.

Using a qualitative multidisciplinary descriptive methodology, we have taken into account economic, social, cultural and recreational aspects by conducting a study of Olympic paddlers, their track record, as well as their participation in the International Sella Descent. The results show a late incorporation of women into the competitive sports field, linked to gender stereotypes, as well as inequalities in participation conditions.

KEYWORDS: Descent of the Sella, canoeing, Spain, women Olympic sport women.

\section{INTRODUCCIÓN}

El Descenso Internacional del Sella fue creado por Dionisio de la Huerta en el corazón de la sociedad rural asturiana a mediados del siglo XX, donde los estereotipos de género femeninos y masculinos estaban totalmente alejados. El deporte, como actividad social se restringía al género masculino, teniendo las mujeres que enfrentarse a los prejuicios de la sociedad que también se reproducían en los medios de comunicación. Esta prueba, que consiguió ser internacional en tan solo quince ediciones guarda similitudes con el festival deportivo por excelencia: los juegos olímpicos. También en esta competición internacional las mujeres han tenido que luchar por hacerse un hueco (Olympic International Committee, 2018).

La revisión bibliográfica nos muestra como la participación de la mujer en los juegos olímpicos ha ido aumentando con el paso de las ediciones. En el caso de la delegación española, los resultados han superado a los masculinos en el medallero olímpico en las últimas competiciones, Londres 2012 y Río 2016 (Olivera Betrán, 2012). Posteriormente nos centraremos en el deporte del piragüismo, en el que las palistas olímpicas, aunque escasas en número han conseguido un palmarés envidiable, siendo el Descenso Internacional del Sella una de las primeras pruebas donde muchas de las deportistas han competido internacionalmente. 


\section{PAPEL DE LA MUJER EN LOS JUEGOS OLÍMPICOS A TRAVÉS DE LA HISTORIA}

La mujer siempre ha estado relegada a un segundo plano en el mundo deportivo. Si nos remontamos a los Juegos Olímpicos de la Antigua Grecia (776 a.C), encontramos como la participación de la mujer en ellos estaba totalmente prohibida, no solo como participante en las competiciones deportivas, sino también como espectadora. Esto no solo ocurría en los Juegos Olímpicos, sino en otros Juegos deportivos como Píticos, Neménicos o Ístmicos. Las mujeres por su parte tenían sus propias competiciones deportivas, los Juegos en honor a la diosa Hera, denominados Juegos Hereos.

En 1896 el barón Pierre de Coubertin funda lo que conocemos como los Juegos Olímpicos modernos y el papel de la mujer, en estos primeros momentos de la reinstauración de los JJOO, es casi inexistente. Son más o menos conocidas declaraciones del barón y de sus ayudantes donde tratan de dejar a la mujer en un segundo plano, como meras acompañantes en la entrega de premios. Pese a estos inicios, lo cierto es que la mujer, con mucho esfuerzo, y sobre todo en los deportes minoritarios, comenzó a participar en las competiciones olímpicas de forma escalonada aunque no en todas las pruebas de un mismo deporte. Por ejemplo, en 1900, en los Juegos de París encontramos la primera participación femenina, de los 997 atletas tan solo 22 eran mujeres. En tenis se proclama campeona olímpica la británica Charlotte Cooper, en 1904 en St. Louis encontramos participación femenina en el tiro con arco y en Estocolmo en 1912 la mujer participa en las pruebas de natación, aunque tan solo pudo hacerlo en los $100 \mathrm{~m}$ y en el relevo $4 \times 100$.

Es a partir de los Juegos de Amsterdan en 1928 cuando la participación femenina comienza a ser más significativa, de los 2606 participantes, 277 eran mujeres. Pese a ello, ser capaces de competir en algunos deportes fue mucho más complejo. En el caso del baloncesto, que fue deporte de exhibición en St. Louis en 1904 y olímpico en Berlín en 1936, el baloncesto femenino no aparece hasta Montreal 1976, es decir, cuarenta años después. Pero incluso hubo que esperar más, ciento ocho años para ser exactos, para la inclusión en el programa del boxeo femenino, pues este deporte es olímpico desde 1904, sin embargo, no lo será en categoría femenina hasta Londres, en el año 2012 (Olympic International Committee, 2018).

Y así la participación femenina fue integrándose en el programa olímpico. La participación española en los Juegos Olímpicos comienza en categoría masculina en los Juegos de París de 1900 con ocho deportistas, las primeras mujeres olímpicas españolas participan veinticuatro años después en el mismo enclave, en los Juegos de París de 1924, fueron dos tenistas. En el caso que nos ocupa, en el piragüismo, este deporte se convierte en olímpico en Berlín en 1936, si bien ya había sido de exhibición en París en 1924, pese a ello, el piragüismo femenino aparece por primera vez en los Juegos Olímpicos de Londres de 1948 (Comité Olímpico Español, 2018).

A la participación olímpica española, sesenta y cuatro años más tarde, en los Juegos Olímpicos de Londres en el año 2012 se les ha denominado las 
"olimpiadas de las mujeres", debido al record de participación femenina en una cita olímpica (Olivera Betrán, 2012). Ciento setenta hombres y ciento doce mujeres formaban el equipo español, y por primera vez en la historia, las mujeres conseguían un mayor número de medallas, de las diecisiete conseguidas, once han sido logradas por mujeres y tan solo seis por hombres. Esta tendencia ha seguido en los Juegos Olímpicos de Río del 2016, de nuevo diecisiete medallas alcanzadas por la delegación española, pero en este caso mucho más igualado, nueve han sido para las mujeres y ocho en categorías masculinas. Los resultados del equipo femenino español son mas significativos si cabe si tenemos en cuenta el porcentaje de mujeres federadas respecto a los hombres, en el año 2017 tan solo el $21.5 \%$ de los deportistas federados eran mujeres (Ministerio de Educación Cultura y Deportes, 2018).

Si bien, la participación femenina ha aumentado de manera significativa desde el comienzo de los Juegos Olímpicos modernos, con una participación en la última edición en torno al $38 \%$ del total de atletas, las condiciones no son las mismas para las atletas femeninas que para sus compañeros masculinos (Juegos Olímpicos y género, 2018). Aparecen diferencias reseñables en el trato que reciben ellas y ellos en los medios de comunicación, exaltando más los triunfos masculinos, o también encontramos diferencias significativas cuando el espectador quiere presenciar el evento deportivo, si lo hacemos en televisión, encontraremos eventos masculinos en el prime time, mientras que los femeninos están relegados a un segundo plano. En el caso que queramos presenciar el evento en directo, las entradas femeninas serán mucho más económicas. Si bien es cierto que actualmente las cuantías económicas por las medallas conseguidas por los y las deportistas son las mismas.

\section{EL PAPEL DE LA MUJER EN EL DESCENSO INTERNACIONAL DEL SELLA}

El Descenso del Sella fue la primera competición piragüística que tuvo lugar en un río en España. En 1929, Dionisio de la Huerta, su fundador, llega a Asturias a veranear desde Barcelona como hacía todos los años en la época estival. Pero en esta ocasión traía una gran novedad, una piragua plegable. Con ella, se aventura por las aguas del río Piloña (afluente del río Sella), acompañado de dos amigos, uno de ellos lo hacía en una piragua de fabricación propia, construida con latas, clavos y un flotador. Animado por esta experiencia se convirtió en una actividad que realizaba cada año modificando el recorrido. Hasta que en 1932, convocó en Arriondas a varios amigos de distintos clubes deportivos de Asturias, la intención de Dionisio era la misma, disfrutar del día, la naturaleza y la compañía de sus amigos haciendo el recorrido Arriondas-Ribadesella, pero su objetivo se vio truncado, cuando muchos de sus invitados quisieron ser los primeros en cruzar el puente riosellano, y así fue como comenzó, ante la oposición de su fundador, el primer descenso competitivo de río en nuestro país(Cuesta, 1994), en la figura 1 Dionisio de la Huerta sostiene uno de los trofeos de la prueba. 


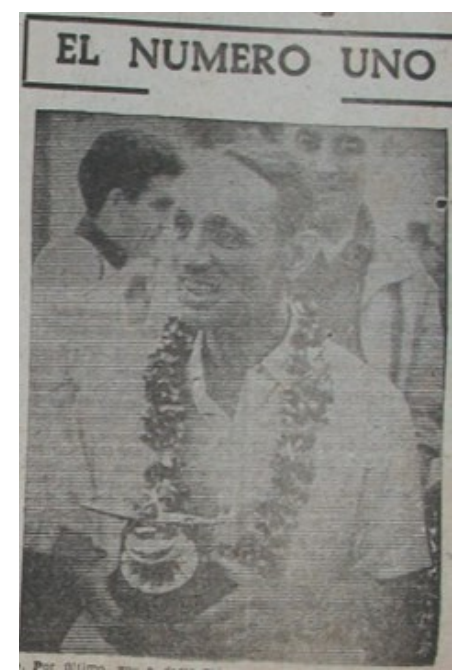

Figura 1. Dionisio de la Huerta en el XXV Descenso Internacional del Sella. (La Región, 06/08/1961).

La prueba fue creciendo, atrayendo cada año a un mayor número de palistas, y también de espectadores, pues Dionisio siempre defendió que el Descenso del Sella era una fiesta antes que una competición. Al mismo tiempo, gracias a sus viajes con la Federación Española de Tenis, promocionaba el Descenso del Sella en distintos países, consiguiendo así que la prueba se convirtiera en internacional en tan solo quince ediciones. En 1951 participaron por primera vez otras naciones, Francia, Italia y Portugal (Blanco Casasola, 1986).

Dionisio se inspiró en las antiguas olimpiadas griegas para forjar, la que durante muchos años fue la prueba deportiva más importante de España. El Descenso se convirtió en su obsesión, hasta el punto, que al día siguiente de finalizar una edición ya estaba ideando elementos para que la siguiente edición fuese más espectacular. Algunos de los aspectos más destacables nos transportan a las antiguas olimpiadas griegas (Martínez, 2012).

El desfile de participantes era uno de estos aspectos, el cual tenía lugar por las calles de Arriondas con los palistas participantes antes de la prueba deportiva. Esta tradición no ha perdurado hasta la actualidad, pues cuando se comenzaron a sobrepasar el millar de participantes, era inviable que todos llegaran a tiempo a la orilla del río para que tuviese lugar la salida. En la actualidad, se realiza un desfile el día anterior al Descenso por la tarde, en la ceremonia de izado de banderas mientras suenan los himnos nacionales de cada país. Posteriormente tiene lugar una salida en verso. Cada año Dionisio de la Huerta los entonaba cual obra del teatro, y tras ellos, sonaba un cañonazo que daba la salida a los miles de palistas allí presentes. En estos versos encontramos claras referencias a los antiguos Juegos Olímpicos, pues los "actos de esta olimpiada", son convocados por un rey, al encontrarnos en Asturias no podía ser otro que el rey Pelayo, además preside los actos un Dios, Neptuno, este Dios, también aparecerá en los diplomas otorgados a los campeones del Sella. Podemos apreciar, por tanto, claras analogías a la mitología griega (Collado Martínez, Robles Tascón, García Morilla y Álvarez del Palacio, 2017).

Tras los casi veinte kilómetros que separan los puentes de Arriondas y Ribadesella, los vencedores eran coronados, con coronas de laurel, árbol 
característico asturiano, en lugar de las tradicionales coronas de olivo griegas. En la actualidad, las coronas de laurel han sido sustituidas por monteras piconas, estos sombreros forman parte del atuendo típico asturiano masculino. Tras tanto esfuerzo, los palistas reponían sus fuerzas con una gran comida que tiene lugar en los campos de Oba, emulando a los antiguos banquetes de los que disfrutaban los olímpicos en la antigüedad. Este lugar de reunión fue fijado por Dionisio en el primer Descenso competitivo, en 1932, tradición que si se mantiene en la actualidad. Sus principios los vemos reflejados en los valores olímpicos: excelencia, buscando siempre la victoria, dando lo mejor de uno mismo, amistad, no solo se fomenta el aspecto competitivo de la prueba, es más se fomenta todo lo que la rodea buscando el disfrute de deportistas y espectadores, y por último, respeto.

¿Qué papel tuvo la mujer en tan singular obra? Hemos analizado varios de los elementos que la alejan al Descenso Internacional del Sella una simple prueba deportiva.

La mujer participa en la prueba desde la primera edición competitiva en 1932. Dionisio, siempre desde la perspectiva inclusiva del deporte, desciendo el Sella en esta primera edición con Marta Junquera. Debemos destacar, como hemos señalado, que la intención de Dionisio no era un descenso competitivo, tal vez por ello vemos como una mujer participa en el primer descenso competitivo de un río en España, en 1932 en el oriente de Asturias, en el corazón de la sociedad rural española. Al año siguiente, otra mujer baja de nuevo junto a Dionisio, en esta ocasión Chona Duran (Prado González, 2006). Durante estas primeras ediciones, también otra mujer realiza el descenso por las aguas del río Sella, la gijonesa Teresa Cerra.

Sin embargo, la participación femenina no se volverá a repetir hasta casi veinte años después, en los años cincuenta. En esta década la participación femenina se restringe a la categoría mixta, en la cual hombres y mujeres descendían conjuntamente de manera recreativa, la categoría mixta competitiva aparecerá posteriormente destacando la participación de parejas mixtas extranjeras. La mujer deportista en Europa comenzaba a verse con regularidad, algo que no sucedía en el rincón oriental asturiano donde tiene lugar la prueba. El contexto social determina esta situación en España, el régimen político en el poder restringía el papel de la mujer al ámbito privado, hogar y familia, mientras que el papel del hombre estaba ligado a la vida pública fuera del hogar (Manrique Arribas, 2018). Las parejas mixtas estaban formadas, como norma general por matrimonios, o por familiares. En 1959, una asturiana, Liliana Martínez de tan solo catorce años baja el río con su hermano de diez, en la prensa de la época describe las críticas a las que fue sometida por realizar esta actividad, reflejo de los estereotipos femeninos, de ellas que se esperaba cualidades como el ritmo, expresividad o flexibilidad y no cualidades ligadas al estereotipo masculino como fuerza o competitividad (López-Crespo y Castejón-Oliva, 2017), cualidades presentes en el piragüismo sobre todo si tenemos en cuenta el medio, pues es una prueba con incertidumbre del espacio en su desarrollo.

Podemos considerar los años sesenta, como una década de contrastes. En 1961 tienen lugar dos hechos reseñables, en primer lugar, aparece la categoría 
damas, por lo que las mujeres comienzan a poder competir. Pero al mismo tiempo, se sigue sin abandonar el concepto mujer/trofeo, pues comienza la tradición de las novias del Sella, la cual consistía en que cada equipo de palistas eligiese a las chicas más hermosas de su lugar de procedencia para alegrar el desfile. La figura número dos (La Voz de Asturias, 08/08/1965), es un claro ejemplo de la visión androcéntrica en los medios de comunicación que reproducen los estereotipos de género, esto provoca que las mujeres deportistas tengan que equilibrar sus habilidades deportivas y supuestas cualidades femeninas como la belleza, para ser aceptadas (Ramírez, Piedra, Ries, y Rodríguez, 2014).

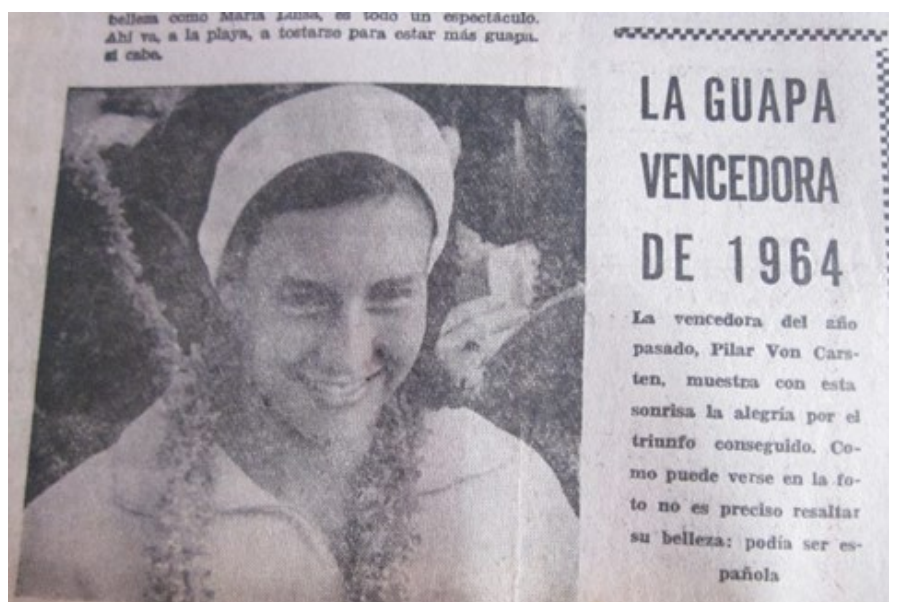

Figura 2. Vencedora del Sella 1964 Pilar Van Carsten (La Voz de Asturias, 08/08/1965).

Si nos centramos en el plano deportivo, en esta primera década competitiva para las mujeres, las palistas internacionales y nacionales se reparten casi de manera equitativa las victorias. Lo cierto es que la participación femenina era muy escasa, en 1968 tan solo ocho mujeres participan en la prueba (La Nueva España, 7/8/1969). En la década de los setenta son seis las palistas nacionales que ganan el Sella frente a cuatro extranjeras. Es en 1978 aparece la primera victoria de palistas asturianas, Margarita Suárez y María del Mar García se proclaman campeonas en K-2 (La Voz de Asturias, 09/08/1978). 


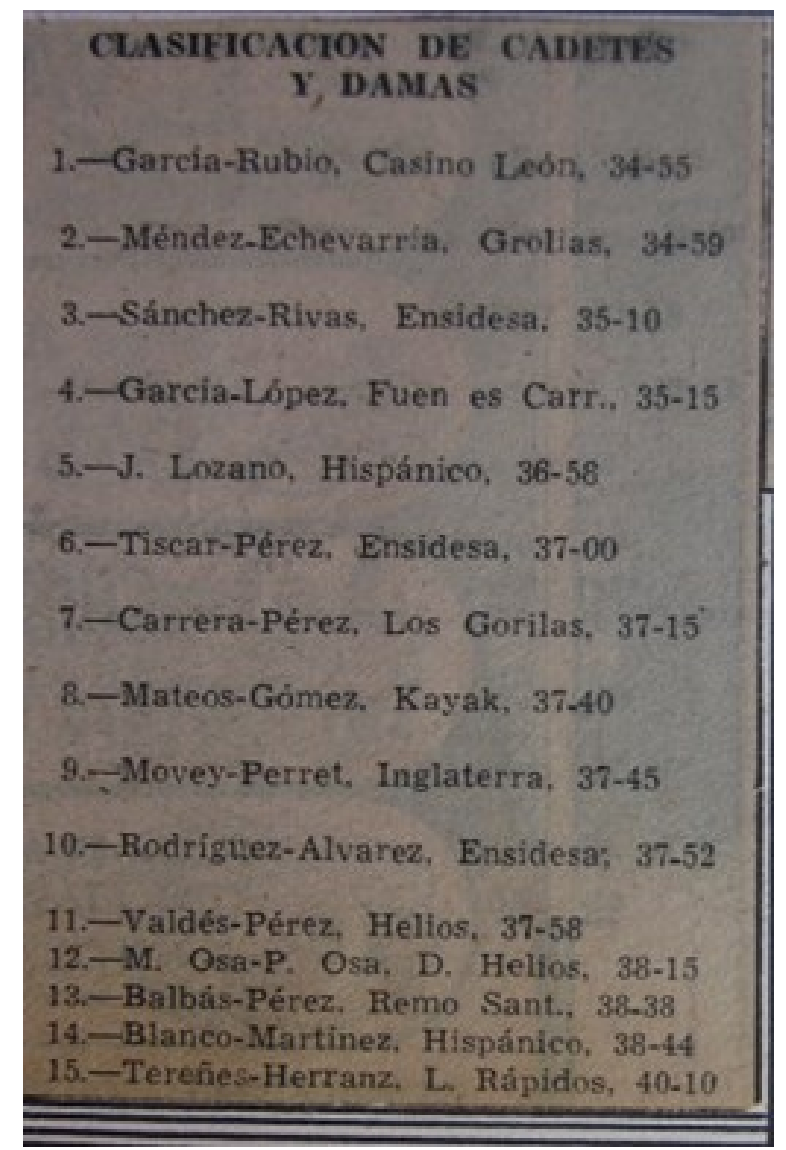

Figura 3. Clasificación Conjunta damas y cadetes 1975 (La Nueva España, 03/08/1975).

En los años ochenta existe una nueva tendencia en la participación femenina, en 1982 hay un aumento significativo de la participación femenina, ciento veintiuno son las palistas que toman la salida (La voz de Avilés, 07/08/1982). Un estudio del archivo del Comité Organizador del Descenso del Sella nos muestra como la mayoría con nacionalidad española. Sin embargo, encontramos entre las vencedoras un número similar de ganadoras nacionales y extranjeras, con doce ganadoras de nacionalidad española y otros ocho triunfos se reparten entre palistas extrajeras en la categoría K-2, en 1980 triunfo norteamericano, en 1981 se hicieron con la victoria palistas de Reino Unido, y un año después en 1982 las primeras mujeres en cruzar el puente de Ribadesella fueron australianas, en 1984 dos nuevas británicas ocuparon el lugar más alto del pódium. En el cambio de década, la victoria de palistas femeninas extranjeras se reduce a tres. En 1990 triunfó el equipo nacional de Polonia, en 1991 la victoria fue para Gran Bretaña y en 1992 para dos palistas austriacas. Es de destacar en esta década el triunfo de la asturiana Almudena Ávila, por primera vez en 1995 junto a Mariam Viña y tres victorias consecutivas en los años 1997,1998 y 1999 junto a Victoria Morazaba.

Con la llegada del siglo XXI, las palistas españolas han dominado el pódium en la categoría K-2, tan solo encontramos tres victorias extrajeras desde el 2000 hasta la actualidad para palistas procedentes de Hungría y para las sudafricanas en el año 2004, el equipo nacional de Sudáfrica repetirá victoria diez años más tarde, en el 2014. De este periodo en la categoría K-2 cabe destacar la embarcación formada por Naiara Gómez-María Pérez ganadoras en tres 
ediciones consecutivas, 2006, 2007 y 2008, posteriormente repetirán victoria en el 2011. María Pérez también se subió a lo más alto del pódium con la leyenda del Sella, Mara Santos en 2009.

En la categoría K-1 la dominadora indiscutible es Mara Santos. La palentina, ostenta el récord de victorias en la prueba asturiana con veintidós victorias, veinte en K-1 y dos en K-2 como hemos visto en el año 2009 junto a María Pérez, y en 2012 junto a María Isabel García, la olímpica que como veremos a continuación más veces ha participado en la prueba asturiana. La leyenda de Mara se forja desde los años ochenta, ganando en las categorías inferiores, cadete e infantil, para posteriormente reinar en la máxima categoría. En el año 2016 recibió el premio Valores del Sella, otorgado por el Comité Organizador del Descenso Internacional del Sella, este emblemático momento está recogido en la figura 4. En el año 2018 fue la elegida para recitar los versos, en la salida de la prueba, con los que emocionaba Dionisio de la Huerta a los miles de personas presentes en Arriondas el primer sábado de agosto después de día dos, como preludio a la espectacular salida.

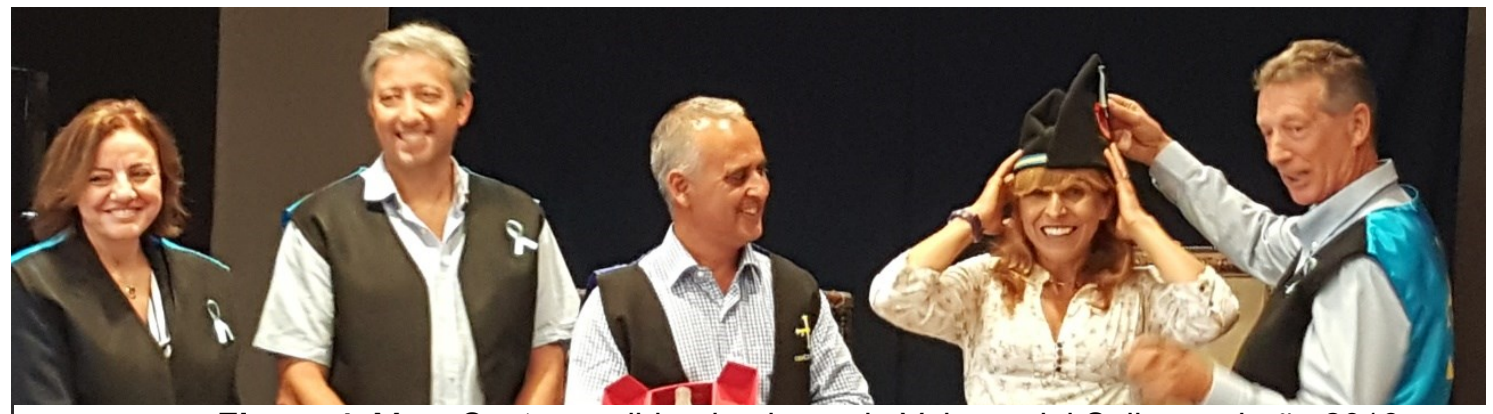

Figura 4. Mara Santos recibiendo el premio Valores del Sella en el año 2016.

(Fotografía colección María Collado).

Es de destacar, para terminar este apartado como Dionisio fue un auténtico visionario, pues como hemos visto promocionó el turismo activo medio siglo antes de que estuviera de moda, haciendo de él, el motor económico del oriente de Asturias en la actualidad. El hizo del deporte su modo de vida y esto ha inspirado a miles de personas, que año tras año visitan el oriente de Asturias para realizar la actividad estrella de turismo activo de la región, bajar el Sella, como lo hacen en competición los profesionales, pero con el objetivo de disfrutar de un día en plena naturaleza con familiares y amigos, tal y como hizo Dionisio con su piragua plegable junto dos amigos hace casi sesenta años (Collado Martínez, 2018).

\section{MUJERES OLÍMPICAS EN EL DESCENSO INTERNACIONAL DEL SELLA}

El Descenso ha sido claramente el propulsor del piragüismo en España, pues gracias a él se fundó la Federación Española de Piragüismo y se fomentó la práctica de esta actividad que ha cosechado un gran número de palistas olímpicos (Antuña Suarez, 2015). Puede que no estuviéramos hablando de mujeres olímpicas, si no hubiese existido el Descenso del Sella pues esta prueba fue la propulsora del piragüismo en nuestro país. Cuando la prueba ya gozaba de rango internacional, aún no existía en España una federación propia, por lo 
que el Descenso del Sella dependía de otra federación, la de remo, lo que en muchas ocasiones se traducía en impedimentos para su desarrollo. Por ello Dionisio de la Huerta, no cesaba de escribir continuas cartas a autoridades deportivas y no deportivas en las que explicaba cómo funcionaba el piragüismo en otros países, estas cartas también iban dirigidas a la Delegación Nacional de Deportes y Jefe de Federaciones. En 1959 se fundó la ansiada Federación Española de Piragüismo, lo que provocó la promoción de diferentes modalidades, así como la formación especializada de técnicos (Cuesta, 1994).

La Federación Española de Piragüismo se funda en 1959, sin embargo, el piragüismo ya era deporte olímpico desde el año 1936 en Berlín y había sido deporte de exhibición en París en el año 1924, lo que permite hacernos una idea del auge que tenía esta práctica deportiva en otros países. El piragüismo masculino español participa por primera vez en unos Juegos Olímpicos en Roma en el año 1960 y las declaraciones de uno de los deportistas españoles resalta la desventaja con la que partía España respecto a otros países. Juan Miguel Feliz fue uno de los representantes españoles, quien había sido ganador del Descenso Internacional del Sella en 1959 junto a José Luis Gutiérrez, describe así su experiencia olímpica "Fue inesperadamente porque claro, era la primera vez que íbamos allá, la experiencia de otras veces en el extranjero lo haciamos fatal y la sorpresa fue que llegamos a semifinales. No lo creíamos" y en la final, "la falta de experiencia, vimos que salían todos como cañones y fuimos detrás de ellos. Y "cascaban" ellos y "cascábamos" nosotros y ahí quedó la cosa" (Radio Televisión Española, 2016).

Por su parte, el piragüismo femenino no participa en unos Juegos Olímpicos hasta los Juegos de Barcelona en 1992. Con la K-4 formada por Luisa María Álvarez Iglesias, Joaquina Costa Iglesias, Ana María Penas Balchada y Belén Sánchez Jiménez y la K-2 formada por Joaquina Costa Iglesias y Belén Sánchez Jiménez (Comité Olímpico Español, 2018). Por tanto, treinta y tres años después de la categoría masculina. No es sorprendente que las palistas españolas tardaran más de tres décadas en lograr participar en unos Juegos si tenemos en cuenta, que el piragüismo español se impulsa gracias al Descenso del Sella, y en este no aparece la categoría de damas hasta 1961, por lo tanto, las mujeres no tenían hueco en la competición hasta ese momento. Hay otros aspectos que también retrasan la participación de la mujer en unos Juegos Olímpicos, que analizaremos a continuación.

\subsection{MUJERES OLÍMPICAS ESPAÑOLAS}

Desde los Juegos Olímpicos de Barcelona en 1992 hasta los Juegos Olímpicos de Río en el año 2016 tan solo diez han sido las palistas que han representado a España, nueve de ellas en aguas tranquilas y una en slalom. Su actuación ha estado muy restringida, pero sus logros han sido reseñables, entre las diez palistas acumulan dos medallas y veintiún diplomas olímpicos.

Una entrevista con una de las cuatro primeras palistas españolas que participaron en unos Juegos representado a España nos ayuda a comprender el porqué de estos datos. Luisa María Álvarez Iglesias, quien en 1996 se convirtió en entrenadora del equipo masculino, nos relata su experiencia en los Juegos de Barcelona. La palista asturiana nos señala como participar en unos Juegos 
Olímpicos es la meta que todo deportista persigue, no siendo comparable con otros eventos internacionales como pueden ser los mundiales de piragüismo, pues en estos solo participan piragüistas. En unos Juegos Olímpicos están concentrados en el mismo lugar todas las estrellas del deporte internacionales, cita por ejemplo las estrellas de la NBA, a nivel organizativo todo está completamente establecido, existe un gran esfuerzo para que todo "salga bien". Otro punto reseñable era el ambiente de la villa olímpica, en la que conviven los deportistas (Álvarez Iglesias, 2018).

Cuando le preguntamos sobre si había algún tipo de discriminación, la respuesta es afirmativa. Una de las principales diferencias es como en categoría masculina, los palistas masculinos eran convocados y estaban concentrados, sin embargo, en la categoría femenina, esto no era así. Esto cambió con la llegada de un nuevo entrenador de origen húngaro, Gyoula Fussesery, quien exigió que el equipo femenino también estuviese concentrado, y con mucho esfuerzo consiguieron clasificarse para vivir el sueño olímpico. Al equipo femenino se le pedían los mayores esfuerzos, las palistas tenían que ganarse su puesto competición tras competición, si los resultados no eran brillantes, no serían convocadas. Cuando llegaba material nuevo, siempre tenía como destinatario el equipo masculino, lo mismo ocurría con las becas, si la subvención era menor, los recortes tendrían lugar en el equipo femenino, incluso suprimiéndose. Todos estos aspectos, junto a estereotipos de género y rol femenino provoca un masivo abandono de las palistas españolas federadas, que en algunas temporadas se sitúa en el $40 \%$ (Isorna Folgar et al., 2019) En la siguiente tabla aparecen las diez olímpicas y sus extraordinarios logros:

Tabla 1. Palistas españolas olímpicas

\begin{tabular}{|c|c|c|c|}
\hline Nombre & $\begin{array}{l}\text { Juegos } \\
\text { Olímpicos }\end{array}$ & Prueba & Clasificación \\
\hline Luisa María Álvarez Iglesias & Barcelona 1992 & Kayak 500 m K4 & Semifinal $4^{\circ}$ \\
\hline Joaquina Costa Iglesias & Barcelona 1992 & $\begin{array}{l}\text { Kayak } 500 \text { m K4 } \\
\text { Kayak } 500 \text { m K2 }\end{array}$ & $\begin{array}{l}\text { Semifinal } 4^{\circ} \\
\text { Semifinal } 9^{\circ}\end{array}$ \\
\hline \multirow[t]{3}{*}{ Ana María Penas Balchada } & Barcelona 1992 & Kayak 500 m K4 & Semifinal $4^{\circ}$ \\
\hline & Atlanta 1996 & Kayak 500 m K4 & $6^{\circ}$ :Diploma \\
\hline & Sydney 2000 & Kayak 500 m K4 & $8^{\circ}$ :Diploma \\
\hline \multirow[t]{3}{*}{ Belén Sánchez Jiménez } & Barcelona 1992 & $\begin{array}{l}\text { Kayak } 500 \text { m K4 } \\
\text { Kayak } 500 \text { m K2 }\end{array}$ & $\begin{array}{l}\text { Semifinal } 4^{\circ} \\
\text { Semifinal } 9^{\circ}\end{array}$ \\
\hline & Atlanta 1996 & $\begin{array}{l}\text { Kayak } 500 \text { m K4 } \\
\text { Kayak } 500 \text { m K1 }\end{array}$ & $\begin{array}{l}6^{\circ}: \text { Diploma } \\
\text { Semifinal } 6^{\circ}\end{array}$ \\
\hline & Sydney 2000 & $\begin{array}{l}\text { Kayak } 500 \text { m K2 } \\
\text { Kayak } 500 \text { m K4 }\end{array}$ & $\begin{array}{l}7^{0} \text { :Diploma } \\
8^{\circ} \text { :Diploma }\end{array}$ \\
\hline \multirow[t]{2}{*}{ María Isabel García Suárez } & Sydney 2000 & Kayak 500 m K2 & $7^{\circ}$ :Diploma \\
\hline & Atenas 2004 & Kayak 500 m K4 & $5^{\circ}$ :Diploma \\
\hline \multirow[t]{4}{*}{ Beatriz Manchón Portillo } & Atlanta 1996 & $\begin{array}{l}\text { Kayak } 500 \text { m K2 } \\
\text { Kayak } 500 \text { m K4 }\end{array}$ & $\begin{array}{l}6^{\circ}: \text { Diploma } \\
6^{0}: \text { Diploma }\end{array}$ \\
\hline & Sydney 2000 & Kayak 500 m K4 & $8^{\circ}$ :Diploma \\
\hline & Atenas 2004 & $\begin{array}{l}\text { Kayak } 500 \text { m K2 } \\
\text { Kayak } 500 \text { m K4 }\end{array}$ & $\begin{array}{l}5^{\circ}: \text { Diploma } \\
5^{\circ} \text { :Diploma }\end{array}$ \\
\hline & Pekín 2008 & $\begin{array}{l}\text { Kayak } 500 \text { m K2 } \\
\text { Kayak } 500 \text { m K4 }\end{array}$ & $\begin{array}{l}\text { Semifinal } 4^{\circ} \\
5^{\circ} \text { :Diploma }\end{array}$ \\
\hline Sonia Molanes Costa & Pekín 2008 & $\begin{array}{l}\text { Kayak } 500 \text { m K2 } \\
\text { Kayak } 500 \text { m K4 }\end{array}$ & $\begin{array}{l}\text { Semifinal } 4^{\circ} \\
5^{\circ} \text { Diploma }\end{array}$ \\
\hline
\end{tabular}




\begin{tabular}{|c|c|c|c|}
\hline \multirow{5}{*}{ Teresa Portela Rivas } & Sydney 2000 & Kayak 500 m K1 & Semifinal $10^{\circ}$ \\
\hline & Atenas 2004 & $\begin{array}{l}\text { Kayak } 500 \text { m K2 } \\
\text { Kayak } 500 \text { m K4 }\end{array}$ & $\begin{array}{l}5^{\circ}: \text { :iploma } \\
5^{\circ}: \text { Diploma }\end{array}$ \\
\hline & Pekín 2008 & $\begin{array}{l}\text { Kayak } 500 \text { m K1 } \\
\text { Kayak } 500 \text { m K4 }\end{array}$ & $\begin{array}{l}\text { Semifinal } 6^{\circ} \\
5^{\circ} \text { Diploma }\end{array}$ \\
\hline & Londres 2012 & Kayak 200 m K1 & $4^{\circ}$ :Diploma \\
\hline & Río 2016 & Kayak 200 m K1 & $6^{\circ}$ :Diploma \\
\hline \multirow[t]{2}{*}{ Jana Smidakova Krompolcova } & Atenas 2004 & Kayak 500 m K4 & $5^{\circ}:$ Diploma \\
\hline & Pekín 2008 & Kayak 500 m K4 & $5^{\circ}$ :Diploma \\
\hline \multirow[t]{3}{*}{ Maialen Chourraut Yurramendi } & Pekín 2008 & Kayak K1 & $16^{\circ}$ Eliminatorias \\
\hline & Londres 2012 & Kayak K1 & $\begin{array}{l}3^{\circ}: \text { Medalla de } \\
\text { Bronce }\end{array}$ \\
\hline & Río 2016 & Kayak K1 & $\begin{array}{l}1^{\circ}: \text { Medalla de } \\
\text { Oro }\end{array}$ \\
\hline
\end{tabular}

\subsection{MUJERES OLÍMPICAS EN EL DESCENSO INTERNACIONAL DEL SELLA}

La participación olímpica de mujeres en el Descenso Internacional del Sella es escasa y está ligada sobre todo a las categorías inferiores como veremos a continuación.

-Luisa María Álvarez Iglesias participa en el Descenso en los inicios de la década de los ochenta, antes de comenzar a competir en campeonatos internacionales. Existían varias razones para ello, la principal causa es porque los campeonatos mundiales suelen ser en agosto, por lo tanto, coincide con la fecha de la prueba deportiva asturiana, que tiene lugar el primer sábado de agosto después del día dos. Otra razón, era como en muchas ocasiones encontraban la oposición de sus entrenadores a participar en la prueba por la posición de salida en la que las mujeres parten en el Sella, pues teniendo que pelear en la salida con mil embarcaciones, que se rompiera la embarcación era habitual, por lo tanto, si bajaban el Sella era con las embarcaciones de peor calidad, claro que tampoco se planteaban ganar, saliendo en una posición tan retrasada simplemente esperabas a ver "que me encuentro por ahí ". Luisa baja en más de una ocasión gracias a que Emilio Llamedo, quien en los años ochenta fuera presidente del Comité Organizador de Descenso Internacional del Sella y presidente de la Federación Asturiana de Piragüismo, le proporcionaba una embarcación apropiada.

-María Isabel García Suárez: esta asturiana es la olímpica que más ha participado en el Descenso. Su participación comienza en la categoría cadete en los años 1993 y 1994, debutando en 1993 con una victoria, posteriormente como junior en 1995 y 1996, en 1996 se hace de nuevo con la victoria. En 1997 debuta en la categoría senior, categoría en la que ha bajado a lo largo de los años tanto en K-1 como en K-2, consiguiendo la victoria en ambas modalidades. Su primera victoria como senior llegó en 1999 en la modalidad K-1 y su última victoria en el año 2012 bajando junto a la llamada reina del Sella Mara Santos, imponiéndose bajo el puente riosellano.

-Jana Smidakova Krompolcova: asturiana de adopción, procedente de la Republica Checa llegó a Asturias con siete años. Participa por primera vez en el 
Descenso del Sella en 1998 en la categoría cadete quedando tercera, al año siguiente, en 1999 consigue el triunfo en la misma categoría. Su última participación la encontramos en el año 2000, esta vez en la categoría junior, categoría en la que también se hace con la victoria.

Como vemos la participación de palistas femeninas del más alto nivel en el Sella es limitada. Podemos nombrar varias causas que pueden ser la clave de esta situación:

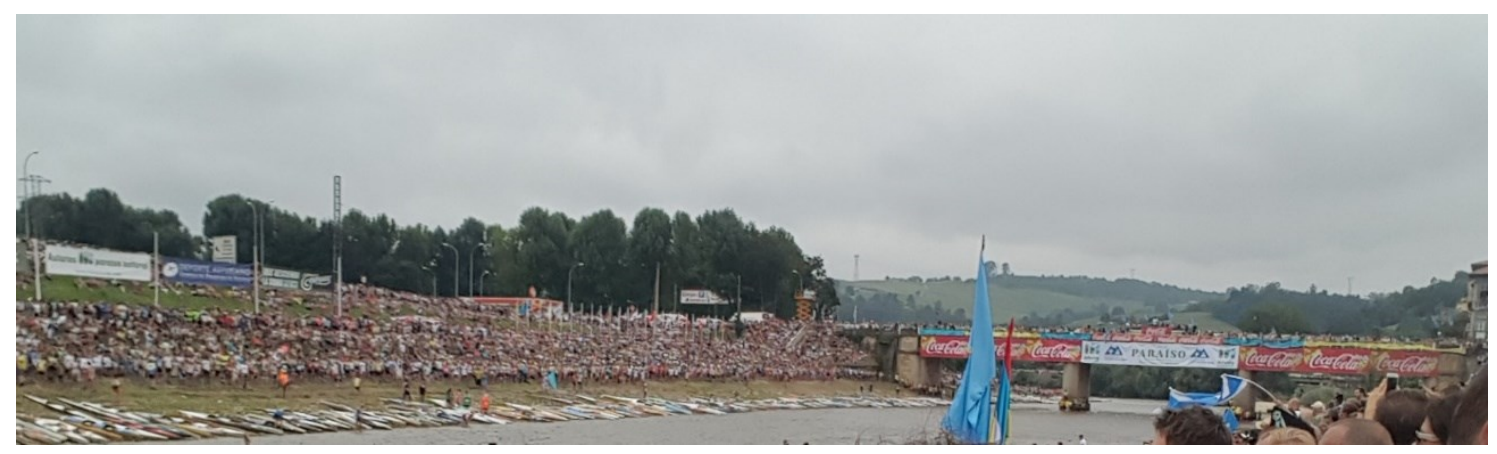

Figura 5. Disposición de las embarcaciones en la salida de Arriondas. (Fotografía colección María Collado).

La primera: el Descenso del Sella es una prueba de larga distancia, y el maratón no es una de las disciplinas que encontramos en el programa a olímpico. Sin duda Mara Santos campeona del mundo de maratón en el año 2000, dos veces subcampeona en el 2002 y 2003 y tres años bronce en el 2001 y 2007, habría sido una gran candidata para aumentar el medallero olímpico español.

La segunda: la posición de salida. ¿Cómo es la salida en el Sella?, partimos de que en la salida no existen igualdad de oportunidades para todos los participantes, el orden de salida es por categorías y es el siguiente: K-2 Senior masculino, K-1 Senior masculino, K-2 Junior masculino, K-1 junior masculino, a partir de aquí irían las mujeres K-2 Senior femenino, K-1 Senior femenino, K-2 Junior femenino, K-1 junior femenino. Y a continuación el resto de las categorías, canoas, cadetes, veteranos y mixtos. ¿Qué implica esto? Vamos a poner como ejemplo la posición de salida de María Isabel García Suarez en el año 1999 donde salió la primera de su categoría K1-DS, ocupaba la posición 566, esto se traduce en más de $200 \mathrm{~m}$ de diferencia con la primera embarcación. En la figura 5 vemos la disposición de las embarcaciones en la salida de la competición junto al puente de Arriondas, a la izquierda de la imagen se situarían los palistas masculinos K-2 Senior y a continuación se comenzarían a situar el resto de categorías siguiendo el orden descrito anteriormente.

¿Qué significa salir en la posición 566?, significa como nos explica Mara Santos (Mara Santos, 2018) que tienes que plantearte la carrera de forma diferente. Lo primero que tienes que definir es el plan o estrategia que vas a seguir, aunque el aspecto clave es la habilidad y capacidad de responder ante cualquier imprevisto. Mara también ha bajado en la categoría mixta junto a su hermano, esta categoría sale aún más retrasada, es significativa cual fue la reacción de su hermano tras la salida jesto es otro mundo!, pues siempre había salido en las primeras posiciones. 
Luisa Álvarez, también nos cuenta su experiencia en la salida, como ya cité "en el Sella no te planteabas ganar, íbamos a ver que nos encontrábamos". Mara nos habla de cómo la suerte es también un factor determinante. Tal vez, eso es lo que le faltó a Luisa en su participación en la prueba, quedó tercera en una ocasión, en las otras dos, rompió embarcación y pala, en una de las ocasiones después de que una K-2 masculina les pasara por encima.

La tercera: discriminación en la entrega de premios. Pues hasta hace dos décadas las mujeres no percibían premios económicos, ni se subían al pódium del Sella que esta situado junto a la meta en el puente de Ribadesella. Si bien es cierto que esto ya no ocurre en las últimas ediciones. Al igual que en otras competiciones internacionales incluidas en Competitions in the World Series (Internacional Canoe Federation, 2018), los ganadores perciben premios económicos, esta establecida una cantidad mínima destinada a estos premios, pero cada competición tiene libertad para distribuirla entre las diferentes categorías (Rodríguez Costales, 2018). En el caso del Sella, desde los años noventa las mujeres también reciben premios económicos y con la llegada del siglo XXI, las cuantías económicas son iguales para la categoría femenina y masculina, además también son coronadas con la montera picona y se suben al pódium junto al puente riosellano.

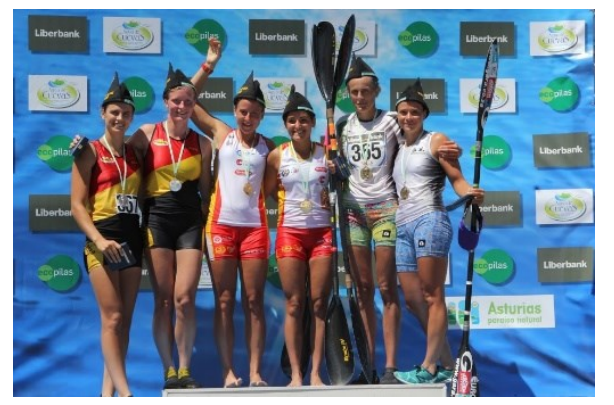

Figura 6. Vencedoras del Sella en su ochenta edición en el podium junto al puente de Ribadesella con la montera picona (El Fielato, 07/0-8/2017).

Todos estos cambios han hecho posible que la tendencia comience a revertirse y en las últimas ediciones hemos visto más participación de palistas femeninas que representan a España internacionalmente como analizaremos a continuación.

\subsection{MUJERES DEL EQUIPO NACIONAL EN EL DESCENSO INTERNACIONAL DEL SELLA}

En la última década podemos encontrar una mayor participación femenina de palistas en el Descenso Internacional del Sella que aspiran a participar en los Juegos Olímpicos, palistas pertenecientes a la selección nacional. Tras un análisis exhaustivo del archivo del Comité Organizador del Descenso Internacional del Sella, algunas de las participaciones más destacadas son: 
-Eva Barrios: participa en el año 2008 en la categoría K-1 senior consiguiendo una tercera posición. En el 2016, de nuevo en la categoría K-1 se queda a las puertas de pódium con una cuarta plaza. En la siguiente edición, en el año 2017, consigue el segundo puesto junto a Aurora Figueras, y en la última edición del Descenso se ha impuesto en la categoría K-2 junto a Amaia Osaba.

-Patricia Coco Rohde: Su primera participación en el Sella es en la categoría cadete en la categoría K-2 junto a Virginia Sánchez en el año 2006 consiguiendo un octavo puesto. En el año 2008, siendo junior, participa en categoría mixta junto a German Sierra llegando en séptima posición. Y en el año 2009 se hace con la victoria en la categoría K-1 junior, en la siguiente edición en el 2010, ya en la categoría senior se queda en el segundo puesto tras Mara Santos. En el año 2012, consigue una octava posición. Su última participación en la prueba asturiana es en el año 2017 consiguiendo la tercera posición en categoría K-2 junto a Cristina Franco.

-Isabel María Contreras Rodríguez: en el año 2016 participa en K-2 junto a Sara Ouzande aunque no finalizan el recorrido. Como hemos analizado anteriormente, ser una de las mejores palistas no está directamente relacionado con conseguir una victoria en el Sella, ni siquiera te garantiza finalizar la prueba. -María Corbera Muñoz: participa en el año 2011 en la categoría mixta consiguiendo un cuarto puesto.

-Tania Fernández García: participa en el año 2017 haciéndose con la victoria en categoría K-1, en el año 2018 consigue la segunda posición en K-2 junto a Tania Elizabeth Álvarez.

-Natalia García Naveiro: participa como junior en el año 2012, junto a Tania Álvarez llegando a la meta en cuarta posición.

-Alicia Heredia Ávila: encontramos su participación en el año 2012 junto a Raquel Bota consiguiendo un séptimo puesto.

-María Begoña Lazcano Guimerans: consigue la segunda posición en categoría mixta junto a lan Carbalo en el año 2016.

-Barbara Pardo Mas: comienza su participación en el Descenso del Sella en la categoría cadete, proclamándose campeona cadete dos años consecutivamente, en los años 2014 y 2015. Posteriormente se hace con la victoria en el año 2016 en la categoría K-2 junior junto a Nora Rey.

-Sara Ouzande Iturralde: como ya hemos visto participa en el año 2016 junto a Isabel María Contreras. Anteriormente participa en categoría mixta en el año 2012 junto a Pablo Claro.

-Ainara Portela: es otra palista que ha comenzado bajando las aguas del Sella desde la categoría cadete en el año 2005 consiguiendo la victoria, más meritorio si tenemos en cuenta que tomó la salida en Arriondas con el dorsal 1082. En la siguiente edición vuelve a competir como cadete revalidando su título de 
campeona cadete. Posteriormente encontramos su participación en el año 2015, llegando a Ribadesella en séptima posición en la categoría K-1 senior.

\section{CONCLUSIÓN}

Desde la antigüedad hasta mediados del siglo $\mathrm{XX}$, el deporte se ha considerado una práctica masculina, ligado a estereotipos de género, del hombre se esperaba ser valerosos, ágil y vigoroso, mientras que el estereotipo de la mujer se relacionaba, con la belleza, gracia y elegancia. Estos estereotipos de género han propiciado la incorporación tardía de la mujer al mundo del deporte. Desde la época clásica, en los Juegos Olímpicos griegos la participación no estaba permitida, existiendo competiciones propias para las mujeres. Con la recuperación de los juegos en el siglo XX, la mujer tampoco pudo incorporarse a la práctica deportiva competitiva en igualdad de condiciones que la categoría masculina.

El Descenso Internacional del Sella, es la competición que hace posible la creación de la Federación Española de Piragüismo, la prueba competitiva aparece en los años treinta, pero la categoría damas no aparece hasta 1961, hasta entonces la participación femenina tenía un carácter recreativo, participando siempre en categoría mixta junto a familiares o esposo. Las primeras palistas que bajaron el Sella tuvieron que hacer frente a los prejuicios de la sociedad tradicional, por no ser considerada una actividad propia de mujeres.

Aunque con aspectos que mejorar el Descenso Internacional del Sella ha ido adaptándose a la nueva era del deporte femenino español. Esta prueba como hemos analizado cuenta con gran participación femenina en categorías inferiores lo que permite ir formando a las palistas en experiencias competitivas internacionales. Sin duda el Descenso Internacional del Sella con todas sus peculiaridades y todos los aspectos que envuelven a la prueba deportiva es algo más que ganar, y a pesar de todas las dificultades que hemos analizado, año tras año las palistas vuelven a las aguas del río Sella.

\section{REFERENCIAS BILIOGRÁFICAS}

Archivo del Comité Organizador del Descenso Internacional del Sella.

Antuña Suárez, A.(2015). Historia del piragüismo olímpico español: la trayectoria deportiva de Herminio Menéndez Rodríguez. Citius, Altius, Fortius Humanismo, sociedad y deporte: Investigaciones y ensayos. Volumen 8 $\mathrm{n}^{\circ} 2$ Noviembre, 2015. ISNN. 2340-9886. Centro de Estudios Olímpicos de la Universidad Autónoma de Madrid. https://doi.org/10.15366/citius2015.8.2

Blanco Casasola, M. (1986). El descenso del Sella y su valor historico deportivo con relación al piragüismo español. Oviedo: Consejería de Educación, Cultura y Deportes del Principado de Asturias.

Collado Martínez, M. (2018). Pervivencia y valor socio-cultural de los juegos y deportes tradicionales en la ribera del rio Sella (Tesis inédita). León: Universidad de León. 
Collado Martínez, M., Robles Tascón, J., García Morilla, S., \& Álvarez Del Palacio, E. (2017). Los valores del Olimpismo en "El descenso del Sella". La relación del creador Dionisio de la Huerta y el Presidente del C. O.I Juan Antonio Samaranch Citius, Altius, Fortius, Humanismo, sociedad y deporte: Investigaciones y ensayos. Volumen $10 n^{\circ} 1$ Junio, 2017. ISSN 2340-9886. Centro de Estudios Olímpicos de la Universidad Autónoma de Madrid. https://doi.org/10.15366/citius2017.10.1.002

Cuesta, J. (1994). Dionisio de la Huerta. Un hombre de tres siglos.Biografía del creador del Descenso del Sella. Gijón: Martecsa.

Comité Olimpico Español. (2 de noviembre de 2018). Deportistas. Disponible en http://www.coe.es/2012/HOMEDEP2012.nsf/2012FDEPORTISTAN2?Op enForm

El Fielato. (07/08/2017). Fotos de todos los pódium del Sella, El Fielato y el Nora. Disponible en http://elfielato.es/deportes/fotos-de-todos-los-podiums-delsella-2783

Internacional Canoe Federation. (4 de noviembre de 2018). Newsletter canoe marathon racing. Disponible en https://www.canoeicf.com/sites/default/files/ 2018-3_cam_newsletter.pdf.

Isorna, M et al. (2019). Mujer y piragua: estudio de las variables moduladoras del abandono deportivo de las mujeres piragüistas en modalidades olímpicas. $\begin{array}{llll}\text { Retos. } & \mathrm{N} & 35 & \mathrm{pp} .\end{array}$ https://recyt.fecyt.es/index.php/retos/article/view/66800

Juegos Olimpicos y género. ( 6 de noviembre de 2018). Historia y Datos de los JJOO y $\quad$ Mujeres. Disponible en http://www.juegosolimpicosygenero.com/p/ historia-de-los-jjoo.html

La Voz de Asturias, 08/08/1965. Diario de Asturias.

La Voz de Asturias, 09/08/1978. Diario de Asturias.

La Voz de Avilés, 07/08/1982. Diario de Avilés

La Nueva España 07/08/1969. Diario de Asturias.

La Nueva España, 03/08/1975. Diario de Asturias.

La Región, 06/08/1961. Diario de Asturias.

López-Crespo, C. y Castejón-Oliva, F.J. (2017). Mujer y pintura deportiva: la colección del Consejo Superior de Deportes (España). Revista Internacional de Medicina y Ciencias de la Actividad Física y el Deporte vol. $17 \quad$ (65) pp. 101-119. Http://cdeporte.rediris.es/revista/revista65/artrepresentacion779.htm. https://doi.org/10.15366/rimcafd2017.65.007

Manrique Arribas, J.C. (2018) La actividad físico-deportiva franquista como intento socializador de la juventud y la mujer. Revista Internacional de Medicina y Ciencias de la Actividad Física y el Deporte vol. 18 (70) pp. 303-329 Http://cdeporte.rediris.es/revista/revista70/artdeporte910.htm. https://doi.org/10.15366/rimcafd2018.70.007

Ministerio de Educación Cultura y Deportes. (25 de noviembre de 2017). Anuario de Estadísiticas Deportivas. Disponible en http://www.mecd.gob.es/redirigeme/?ruta =/ser vicios -al-ciudadano$\mathrm{mecd} / \mathrm{dms} / \mathrm{mecd} / \mathrm{servicios}$ - al-ciudadano mecd/estadisticas/deporte /anuario-deporte/AED-2017/Anuario_de_Estadisticas_Deportivas _2017.pdf 
Martínez, P. (31 de Julio de 2012). "El origen olímpico del Sella" entrevista a José Manuel Zapico, La Nueva España. Disponible en http://www.Ine.es/verano/2012/07/31/origen-olimpico-sella/1278100.html

Olivera Betrán, J.(2012). Juegos Olímpicos Londres 2012: la olimpiada de las mujeres. Apunts. Educación Física y Deportes. n. ${ }^{\circ} 109$, 3.er trimestre (julio-septiembre), $\quad$ pp. 7-10. ISSN-1577-4015. https://doi.org/10.5672/apunts.2014-0983.es.(2012/3).109.00

Olympic International Committee. (22 de noviembre de 2018). Olympic Games. Disponible en https://www.olympic.org/

Prado González, R. (2006). 70 años del Descenso del Sella. Gijón: VTP Editorial.

Radio Televisión Española. (9 de agosto de 2016). Conexión Vintage. Descenso Internacional del Sella. Disponible en http://www.rtve.es /alacarta/videos/conexion-vintage/conexion-vintage-descensointernacional-del-sella/3676180/

Ramírez, G.; Piedra, J.; Ries, F. y Rodríguez, A.R. (2014). La mujer y el deporte en el cine del siglo XXI (2000-2009). Revista Internacional de Medicina y Ciencias de la Actividad Física y el Deporte vol. 14 (56) pp. 719-734 Http://cdeporte.rediris.es/revista/revista56/artABFR506.htm

\section{FUENTES ORALES}

Luisa María Álvarez Iglesias. Primera palista olímpica española en Barcelona 1992. Entrevista realizada por la autora en noviembre de 2018.

Mara Santos García. Campeona del Sella en veintidós ocasiones. Entrevista realizada por la autora en noviembre de 2018.

Begoña Rodríguez Costales. Miembro del Comité de Maratón de la Federación Internacional. Entrevista realizada por la autora en noviembre de 2018.

Número de citas totales / Total references: 30 (100\%)

Número de citas propias de la revista / Journal's own references: $3(10 \%)$

Rev.int.med.cienc.act.fís.deporte - vol. 21 - número 81 - ISSN: 1577-0354 